\title{
Carrier recombination effects in strain compensated quantum dot stacks embedded in solar cells
}

\author{
D. Alonso-Álvarez, ${ }^{1, a)}$ A. G. Taboada, ${ }^{1}$ J. M. Ripalda, ${ }^{1}$ B. Alén, ${ }^{1}$ Y. González, ${ }^{1}$ \\ L. González, ${ }^{1}$ J. M. García, ${ }^{1}$ F. Briones, ${ }^{1}$ A. Martí, ${ }^{2}$ A. Luque, ${ }^{2}$ A. M. Sánchez, ${ }^{3}$ and \\ S. I. Molina ${ }^{3}$ \\ ${ }_{1}^{1}$ Instituto de Microelectrónica de Madrid, CNM (CSIC), c/Isaac Newton 8, PTM, Tres Cantos, \\ 28760 Madrid, Spain \\ ${ }^{2}$ Instituto de Energía Solar (IES), UPM, Ciudad Universitaria, 28040 Madrid, Spain \\ ${ }^{3}$ Departamento de Ciencia de los Materiales e Ing. Metalúrgica y Q. I. Universidad de Cádiz, \\ Campus Universitario de Puerto Real, Puerto Real, 11510 Cádiz, Spain
}

(Received 16 July 2008; accepted 15 August 2008; published online 25 September 2008)

\begin{abstract}
In this work we report the stacking of $50 \mathrm{InAs} / \mathrm{GaAs}$ quantum dot layers with a GaAs spacer thickness of $18 \mathrm{~nm}$ using GaP monolayers for strain compensation. We find a good structural and optical quality of the fabricated samples including a planar growth front across the whole structure, a reduction in the quantum dot size inhomogeneity, and an enhanced thermal stability of the emission. The optimized quantum dot stack has been embedded in a solar cell structure and we discuss the benefits and disadvantages of this approach for high efficiency photovoltaic applications. () 2008 American Institute of Physics. [DOI: 10.1063/1.2978243]
\end{abstract}

Quantum confinement of carriers in quantum dots (QDs) has been proposed as a possible means to materialize the intermediate band solar cell and hot exciton extraction concepts. ${ }^{1-3}$ The objective is to increase the short circuit current without decreasing the open circuit voltage of the devices. Due to the small volume of the nanostructures, their contribution to the total photocurrent is generally small, so stacks of tens of QD layers are usually needed. However, this is well known to also increase the accumulated stress of the material during growth generating dislocations and nonradiative recombination centers. ${ }^{4}$ Introduction of strain compensation (SC) to reduce the accumulated stress is a promising way of improving the material quality and the efficiency of devices based on QDs. ${ }^{5,6}$ Several materials have been used for $\mathrm{SC}$ of the InAs/GaAs QD system such as $\mathrm{GaP}^{5}{ }^{5} \mathrm{GaInP},{ }^{7}$ or GaNAs. ${ }^{8}$ In this work we report the stacking of $50 \mathrm{InAs}$ QD layers using $2 \mathrm{GaP}$ ML for SC and a stack period of only $18 \mathrm{~nm}$. We preferred a "digital" SC by the introduction of single monolayer of GaP instead of a GaAsP alloy due to the difficulty to control the phosphorus to arsenic incorporation ratio during growth of the stacked QD structure.

Our samples were designed to meet the zero-stress condition as stated by Ekins-Daukes et al. ${ }^{9}$ under the following assumptions: (a) the InAs and $\mathrm{GaP}$ layers grow pseudomorphically on GaAs, (b) the layer thicknesses do not depend on the in plane strain $[1 \mathrm{ML}(\mathrm{GaP})=0.273 \mathrm{~nm}$ and $1 \mathrm{ML}(\mathrm{InAs})=0.303 \mathrm{~nm}$ regardless of the in plane strain], and (c) there is only biaxial stress during growth. The calculation predicts a ratio of $0.9 \mathrm{ML}$ of GaP to $1 \mathrm{ML}$ of InAs to fully compensate the stress. However, we round up this quantity to $1 \mathrm{ML}$ in an attempt to compensate for the low incorporation ratio of phosphorus in GaAs. The QD stack was grown by molecular beam epitaxy (MBE) at $510{ }^{\circ} \mathrm{C}$ on GaAs (001). For each QD layer, $2 \mathrm{ML}$ of InAs were deposited at a low growth rate $(0.021 \mathrm{ML} / \mathrm{s})$ to improve QD size homogeneity, ${ }^{10}$ with $1 \mathrm{ML}$ of GaP $1.53 \mathrm{~nm}$ below and an-

${ }^{a)}$ Electronic mail: diego@imm.cnm.csic.es. other one $12.6 \mathrm{~nm}$ above as shown in Fig. 1(a). The top GaP layer was placed farther from the QDs than the bottom one to avoid the formation of an undesirable InP phase due to In outdifussion. We used $1 \mathrm{ML}+1 \mathrm{ML}$ of GaP instead of just $2 \mathrm{ML}$ of $\mathrm{GaP}$ to keep the barriers as thin as possible and facilitate the tunneling of carriers through them. The spacer layer thickness $(\mathrm{GaAs}+\mathrm{GaP})$ between QD layers was $18 \mathrm{~nm}$. The critical thickness for QD nucleation, as deduced from the two-dimensional to the three-dimensional (3D) transition

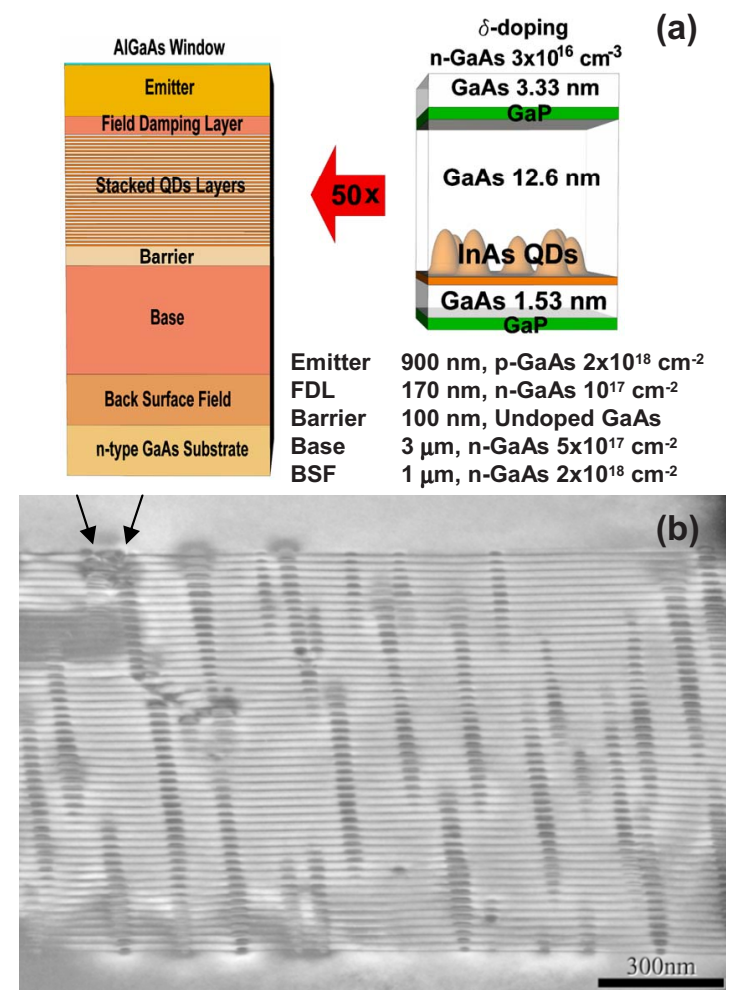

FIG. 1. (Color online) (a) Structure of the 50 stacked QD solar cell with SC layers. Each GaP layer has a nominal thickness of $1 \mathrm{ML}=0.273 \mathrm{~nm}$. (b) Cross-sectional TEM image of the 50 stacked QD layers. The arrows indicate the defects observable in the image. 


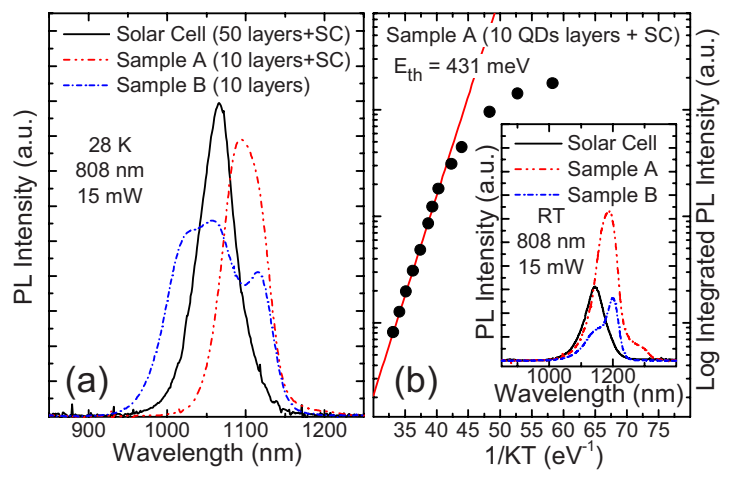

FIG. 2. (Color online) (a) Low temperature PL at $808 \mathrm{~nm}$. b) Evolution of the integrated PL intensity of sample A with temperature. The inset shows the comparison of the RT PL of the three samples.

in the reflection high energy electron diffraction (RHEED) measurements, was very stable for the 50 QD layers of the stack at $1.62 \pm 0.04 \mathrm{ML}$. On the contrary, no 3D transition can be observed beyond the fourth layer in a reference sample without SC due to the blurred RHEED signal as a consequence of the increased surface roughness. ${ }^{11}$ With the aim of controlling the amount of SC, we began each $\mathrm{GaP}$ monolayer by first depositing 1.2 ML of Ga on the $(2 \times 4)$ As rich reconstruction with the As cell fully closed (we use a custom built As cell), and then opening the $\mathrm{P}$ cell at a beam equivalent pressure of $P_{2}=2.3 \times 10^{-7}$ Torr for $2.7 \mathrm{~s}$. The results of the $\mathrm{x}$-ray characterization of the grown samples are compatible with the incorporation of $1 \mathrm{ML}$ of $\mathrm{GaAs}_{0.6} \mathrm{P}_{0.4}$ per each nominal GaP monolayer (equivalent to a $44 \%$ of SC). This 50 QD stack layers are further introduced in a solar cell structure. The complete epitaxial layer sequence of the solar cell is shown in Fig. 1(a). Standard optical lithography and wet etching techniques were used to define mesas and metal contacts.

A representative cross-sectional transmission electron microscopy (TEM) image is shown in Fig. 1(b). The image reveals that the structure is almost defect-free with some defects in the very last few layers (indicated by arrows in the image). These defects seem to be produced by the interaction of very close or especially large QDs. The image also reveals columnar growth of the QDs along the structure. We measure in the image a tilt for the columns of $7^{\circ}$ with respect to the growth direction. This kind of growth suggests the existence of strain around the nanostructures that propagates to the following layer, creating spots of increased nucleation probability. Moreover, it has been reported that a certain asymmetry in the composition of the stacked nanostructures leads to the tilting of the columns. ${ }^{12,13}$

Figure 2(a) shows the photoluminescence (PL) spectra of the solar cell structure recorded at $30 \mathrm{~K}$. The other two spectra correspond to simplified epitaxies containing only $10+1$ (uncapped) stacked QD layer growth in the same conditions but with (sample A) and without (sample B) SC layers. Up to three Gaussian contributions can be clearly observed in sample B, at 1.11, 1.17, and $1.21 \mathrm{eV}$. The analysis of the excitation power dependence and the atomic force micrographs of the uncapped layer (not shown) suggests a broad multimodal distribution of QD sizes in this sample. On the other hand, a much narrower spectrum characterizes the emission of the solar cell and sample A, with peak energies at 1.16 and $1.13 \mathrm{eV}$, respectively. Although the full width at
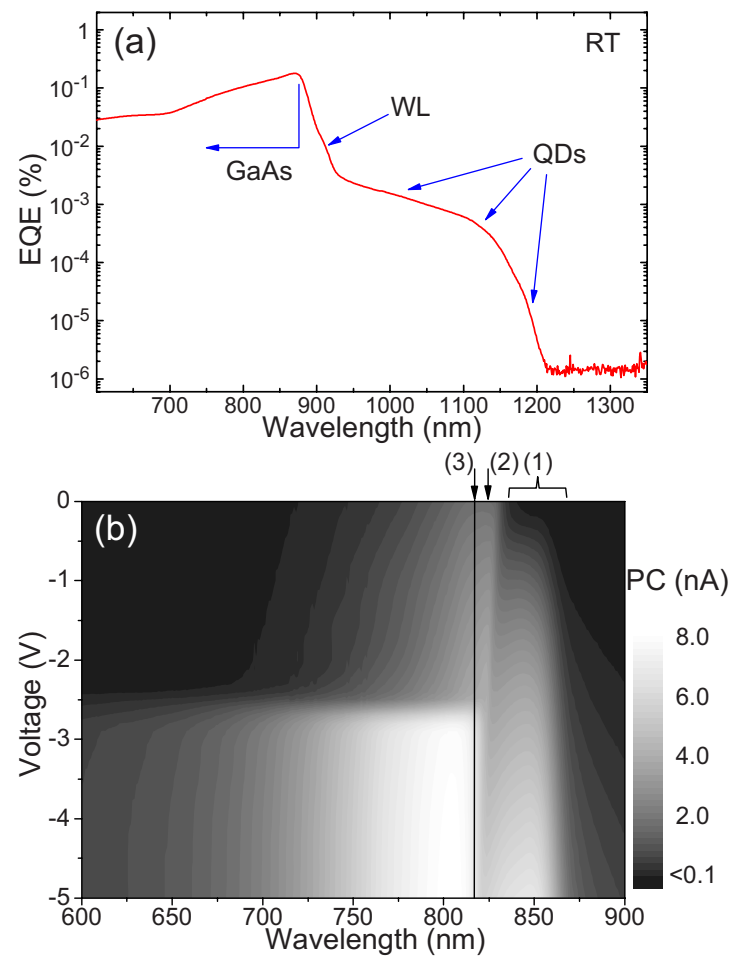

FIG. 3. (Color online) (a) EQE at RT. (b) Photocurrent measurements at low temperature as a function of the applied bias.

half maximum of $60 \mathrm{meV}$ is still too large to consider a monomodal distribution in all layers, our results clearly demonstrate the improvement of the material quality as a consequence of the introduction of GaP layers. The solar cell intensity cannot be compared here given the different capping layers present in this case. It should be noticed that the PL emission of the solar cell is blueshifted by $30 \mathrm{meV}$ with respect to sample A despite having the same nominal structure. We believe that this is due to sample annealing during the growth of the solar cell emitter at $580{ }^{\circ} \mathrm{C}$. It is well known that this annealing favors the In-Ga interdifussion between the dots and the matrix increasing the PL emission energy as observed. ${ }^{14} \mathrm{~A}$ similar blueshift has been obtained by annealing sample A in the same conditions used for the epitaxial growth of the emitter. The three samples exhibit room temperature (RT) PL as shown in the inset of Fig. 2(b), the PL intensity of sample A being 2.4 times more intense than that of sample B. Moreover, the temperature dependence of the emission spectrum reveals an additional consequence of the SC process. As shown in Fig. 2(b), an Arrhenius-type plot of the integrated PL intensity yields a carrier thermal activation energy for sample A of $431 \mathrm{meV}$, double than that obtained for standard InAs/GaAs QDs. ${ }^{15} \mathrm{In}$ view of our results, the GaP incorporation in the matrix results in a decreased number of nonradiative defects around the dots and an enhanced thermal stability with great interest in light detection and emission applications of this kind of nanostructures.

We have evaluated the performance of our structure as a solar cell measuring its external quantum efficiency (EQE). As shown in Fig. 3(a), the QD solar cell has an extended spectral response of up to $1.2 \mu \mathrm{m}$ due to interband light absorption in the nanostructures. However, our results also indicate a very poor response of the device in the short wavelength range. Several effects may contribute to this reduction 
in the above gap photocurrent. First, it is well known that solar cells' blue response is greatly affected by recombination of carriers at the surface of the structure. Passivation treatments and thin GaInP or AlGaAs barriers are commonly used to reduce the recombination rate and to keep the electrons away from the surface. In the design of this cell we chose AlGaAs as the barrier material, but its tendency to oxidize, especially in $\mathrm{Al}$ rich alloys may have canceled its benefits. Second, although the TEM image and the PL measurements indicate good structural quality, defects present in the last few layers of QDs in the field damping layer (FDL) and in the emitter region may act as nonradiative recombination centers and traps for electrons. Since we only achieved partial SC according to the x-ray diffraction measurements, this problem could be solved in the future by increasing the GaP content of the sample to the estimated optimum value. Finally, carrier lifetime in the QDs is smaller than that in bulk GaAs, meaning, the minority carriers that have to cross the junction through the QD region will tend to recombine instead of being collected in the base and the emitter. Moreover, such undesirable recombination effect in the QDs could be enhanced by two factors: (a) the GaP barriers that prevent the free movement of carriers and (b) the low electric field in the region due to the screening of the FDL. At RT the impact of the former in the photocurrent should be minimum, taking into account the small thicknesses of the GaP barriers. The latter could be avoided simply by removing the FDL. This was originally included in the design to keep the QDs of the whole stack in the same state of charge and allow the formation of a half-filled intermediate band. However, if stacking a very large number of QD layers is possible, this condition is automatically satisfied by most of them and the FDL would not be necessary. ${ }^{16}$ Finally, to further investigate the effect of the nanostructures in the EQE, we have performed photocurrent measurements of the solar cell at $16 \mathrm{~K}$ as a function of the applied bias. Two distinct evolutions can be observed in Fig. 3(b). First, illuminating below the GaAs gap at the wetting layer (WL) wavelength [region labeled as (1)], the photocurrent increases smoothly with the reverse bias as more carriers contribute to the signal by tunneling through the WL and the GaP barriers. ${ }^{17}$ A similar evolution is found for the contribution labeled as (2) corresponding to absorption in the GaAs spacer layer between QDs where some amount of In has been incorporated as a consequence of the unintentional annealing stated before. The position of the absorption edge coincides with the expected edge position for an InGaAs alloy with $1.5 \%$ In. On the other hand, illuminating above the GaAs edge, labeled as (3), the photocurrent exhibits a sudden increase at around $-2.5 \mathrm{~V}$, becoming almost constant after that point. This increase is observable for the whole wavelength range above the GaAs edge. Since most of the short wavelength light is absorbed in the emitter, we believe this is indicative of the presence of defects in the upper part of the cell that act as electron traps. An increase in the field allows the ionization of deep level centers contained in the FDL until the signal saturates when all available traps are ionized. ${ }^{18}$ Light with a wavelength below the GaAs edge and absorbed in the QD region is not affected by this phenomenon, as observed in Fig. 3(b).

In conclusion, we have studied the effect of digital SC with GaP in samples containing up to 50 stacked InAs/GaAs QD layers grown by MBE. Good structural and optical qualities have been found by the analysis of TEM images and PL spectra. The GaP barriers produce an excellent thermal stability of the optical properties of the QD stacks by increasing the activation energy for carrier escape. Furthermore, the optimized QD stack has been embedded in a solar cell structure. Extended absorption has been observed beyond the GaAs edge due to the nanostructures together with a poor blue response of the cell. This is partially ascribed to the particular solar cell structure design, but also due to the high carrier recombination in the QDs and trapping by defects associated to the introduction of the QD layers. Further work must be done to fully understand this phenomenon and the benefits and disadvantages of SC methods in photovoltaic applications using nanostructures.

The authors gratefully acknowledge financial support by the Spanish MEC and CAM through Projects 200560M089, S-05050/ENE-0310, TEC-2005-05781-C0301, and -02, Consolider-Ingenio 2010 CSD2006-0004, the Junta de Andalucia (Project TEP383, Group TEP120), and by the European Commission through the SANDIE Network of Excellence (NMP4-CT-2004-500101).

\footnotetext{
${ }^{1}$ A. Luque and A. Martí, Phys. Rev. Lett. 78, 5014 (1997).

${ }^{2}$ A. Martí, L. Cuadra, and A. Luque, Conference Record of the 28th IEEE Photovoltaic Specialists Conference-2000, 2000 (unpublished), pp. 940943.

${ }^{3}$ A. J. Nozik, Physica E (Amsterdam) 14, 115 (2002).

${ }^{4}$ J. T. Ng, U. Bangert, and M. Missous, Semicond. Sci. Technol. 22, 80 (2007).

${ }^{5}$ J. Tatebayashi, N. Nuntawong, Y. C. Xin, P. S. Wong, S. H. Huang, C. P. Hains, L. F. Lester, and D. L. Huffaker, Appl. Phys. Lett. 88, 221107 (2006).

${ }^{6}$ R. B. Laghumavarapu, M. El-Emawy, N. Nuntawong, A. Moscho, L. F. Lester, and D. L. Huffaker, Appl. Phys. Lett. 91, 243115 (2007).

${ }^{7}$ N. Nuntawong, S. Birudavolu, C. Hains, S. Huang, H. Xu, and D. Huffaker, Appl. Phys. Lett. 85, 3050 (2004).

${ }^{8}$ R. Oshima, T. Hashimoto, H. Shigekawa, and Y. Okada, J. Appl. Phys. 100, 083110 (2006).

${ }^{9}$ N. Ekins-Daukes, K. Kawaguchi, and J. Zhang, Cryst. Growth Des. 2, 287 (2002).

${ }^{10}$ M. Lachab and H. Sakaki, Appl. Surf. Sci. 254, 3385 (2008).

${ }^{11}$ K. Koike, S. Shuwei, and M. Yano, Jpn. J. Appl. Phys., Part 1 39, 1622 (2000).

${ }^{12}$ B. Alén, J. Martínez-Pastor, L. González, J. M. García, S. I. Molina, A. Ponce, and R. García, Phys. Rev. B 65, 241301 (2002).

${ }^{13}$ S. I. Molina, T. Ben, D. L. Sales, J. Pizarro, P. Galindo, M. Varela, S. Pennycook, D. Fuster, Y. González, and L. González, Nanotechnology 17 , 5652 (2006)

${ }^{14}$ J. Tatebayashi, Y. Arakawa, N. Hatori, H. Ebe, M. Sugawara, H. Sudo, and A. Kuramata, Appl. Phys. Lett. 85, 1024 (2004)

${ }^{15}$ J. M. Ripalda, D. Alonso-Álvarez, B. Alén, A. G. Taboada, J. M. García, Y. González, and L. González, Appl. Phys. Lett. 91, 012111 (2007).

${ }^{16}$ A. Martí, N. López, E. Antolin, E. Canovas, C. Stanley, C. Farmer, L. Cuadra, and A. Luque, Thin Solid Films 511, 638 (2006)

${ }^{17}$ B. Alén, J. Bosch, D. Granados, J. Martínez-Pastor, J. M. García, and L. González, Phys. Rev. B 75, 045319 (2007).

${ }^{18}$ P. Rodin and I. Grekhov, Appl. Phys. Lett. 86, 243504 (2005).
} 